\title{
Modelo de elementos finitos para el análisis térmico de estructuras hormigonadas por etapas
}

\author{
Finite element model for thermal analysis of structures concreted in stages
}

Fecha de entrega: 29 de julio 2014

Fecha de aceptación: 23 de septiembre 2014

\section{José I. Croppi y M. Cecilia Lazzaroni}

Facultad Regional Santa Fe, Universidad Tecnológica Nacional, Lavaise 610 - S3004EWB Santa Fe, Argentina, jcroppi@frsf.utn.edu.ar, mlazzaroni@frsf.utn.edu.ar

La distribución de temperaturas en el interior de una estructura de hormigón masivo a edades tempranas, es un fenómeno que debe ser estudiado y caracterizado, ya que el calor liberado durante la construcción por efectos de la reacción de hidratación del cemento, puede originar tensiones inadmisibles para su edad, produciendo fisuras y disminución de resistencia. El objetivo general del trabajo consiste en proponer un método numérico que permita determinar el estado térmico del hormigón al comienzo de la hidratación del cemento, y poder aplicar esta metodología al estudio de estructuras civiles con gran volumen de hormigón. Esto se logra resolviendo la ecuación diferencial del calor mediante el método de los elementos finitos, agregando un término fuente que represente la generación del calor y, a su vez, estableciendo una condición de convección en las superficies expuestas al aire para poder estudiar el efecto de la variación de la temperatura ambiente en el tiempo. El modelo se valida a través de la comparación de resultados con ensayos experimentales sobre probetas de pasta de cemento y de hormigón, con resultados de la bibliografía y con soluciones obtenidas mediante programas comerciales.

Palabras claves: estructuras de hormigón masivo, hidratación del cemento, métodos numéricos, elementos finitos
The temperature field inside a massive early aged concrete structure should be carefully studied and described, because the heat released during the hydration of the cement reaction can induce an unacceptable level of stresses. These stresses may lead to the concrete cracking and strength loss. The main objective of this paper is to propose a numerical method capable of evaluating the thermal state of concrete during the cement hydration reaction, and to be able to apply this methodology to the analysis of massive concrete structures. This simulation can be achieved by solving the differential heat equation using the Finite Element Method, adding a source term that represents the heat generation and, at the same time, setting a convection condition on the surfaces exposed to the air in order to study the variation in time of the environmental temperature. This model is properly validated by comparing with experimental results in concrete specimens, results from bibliography and numerical solutions obtained using commercial softwares.

Keywords: massive concrete structures, cement hydration, numerical methods, finite elements.

\section{Introducción}

Debido al proceso de hidratación del cemento, sumado a la baja conductividad del material y al método rápido de construcción, aparecen gradientes térmicos elevados entre el interior de la masa y la superficie exterior en estructuras de hormigón masivo. Durante el enfriamiento, debido a la presencia de restricciones interiores y exteriores, como por ejemplo las fundaciones, este gradiente térmico causa tensiones térmicas importantes. Si estas tensiones térmicas, sumadas a otras solicitaciones debido a cargas externas, 
exceden la resistencia del hormigón, se producirán fisuras que atentan contra la durabilidad estructural (e.g., Azenha y Faria, 2008; Crespo, 2011). Es por esto que un análisis térmico cumple un rol muy importante en el diseño y la construcción de este tipo de estructuras. Recientemente se ha notado un creciente interés en el diseño y construcción de presas de hormigón en Argentina. Surge entonces la idea de contar con una herramienta capaz de simular numéricamente la evolución de temperaturas en este tipo de estructuras, que permita diseñar un hormigonado por etapas mediante alguna estrategia análoga a las presentadas en Cervera et al. (1999) y Jaafar et al. (2007), facilitando la optimización en la planificación de las etapas constructivas, y la aplicación del modelo para cualquier estructura en general.

El objetivo de este trabajo es desarrollar e implementar un modelo numérico por elementos finitos para el cálculo de la evolución de temperaturas en dos y tres dimensiones, mediante el cual sea posible tener en cuenta factores como: la generación de calor debido a la reacción de hidratación del cemento, los múltiples materiales involucrados (hormigón colocado con anterioridad, encofrados, suelo o aislaciones, etc.), la variación térmica del entorno donde se emplaza la estructura, las etapas y tiempos de ejecución de las mismas, entre otros.

\section{Metodología numérica}

La formulación teórica del problema de generación y conducción de calor en el hormigón que se produce durante la hidratación del cemento, conduce a un problema en derivadas parciales transitorio no lineal, al que se le aplican condiciones iniciales y de contorno (Incropera y DeWitt, 2002).

El problema de conducción de calor transitorio con término fuente en un medio continuo que abarca un dominio $\Omega$, está gobernado por la siguiente ecuación diferencial (Sergerlind, 1984):

$$
\rho c_{e} \dot{\phi}-k \Delta \phi=\dot{Q} \text { en } \Omega
$$

donde $\Delta$ es el operador laplaciano, $\phi$ es el campo de temperaturas y los parámetros $\rho, c_{e}$ y $k$ son la densidad, el calor específico y la conductividad térmica, respectivamente, que dependen del material. El término fuente es representado por $\dot{Q}$, el cual es función del grado de hidratación del cemento. Las etapas constructivas son activadas y consideradas en el análisis según tiempos preestablecidos. A su vez, se introduce una interfaz de contacto entre las distintas etapas, para contar con la posibilidad de establecer una condición de convección o una condición de conductancia.

\section{Condiciones de borde e iniciales}

Las condiciones de borde se describen en términos de las ecuaciones siguientes:

$$
\begin{aligned}
& \phi=\bar{\phi} \text { en } \Gamma_{\phi} \\
& -k \frac{\partial \phi}{\partial n}=\bar{q} \cdot \bar{n} \text { en } \Gamma_{n} \\
& \bar{q} \cdot \bar{n}=h\left(\phi-\phi_{r e f}\right)
\end{aligned}
$$

donde $\Gamma_{\phi}$ y $\Gamma_{n}$ son las fronteras del dominio $\Omega$ de tipo Dirichlet (2) y de tipo Neumann (3), definidas de manera tal que $\Gamma_{\phi} \cup \Gamma_{n}=\Gamma \mathrm{y} \Gamma_{\phi} \cap \Gamma_{n}=\varnothing, \bar{\phi}$ representa temperaturas impuestas, $\bar{n}$ es un vector normal a la frontera $\Gamma$ y $\bar{q}$ son flujos en la frontera tipo Neumann (3). El flujo térmico entre el sólido y el exterior se modela mediante (4) que representa una condición de convección. Allí interviene el coeficiente de convección $h$, la temperatura de cálculo $\phi$ en la superficie y una temperatura de referencia conocida $\phi_{\text {ref }}$, mediante la cual se tiene en cuenta la influencia de la temperatura ambiente exterior, variable en el tiempo. Las condiciones iniciales de cada etapa se establecen mediante:

$\phi(x, t=0)=\phi_{0}(x)$ en $\Omega$

donde $\phi_{0}(x)$ representa la distribución inicial de temperaturas que en general coincide con la temperatura de colado de cada etapa.

\section{Término fuente de generación de calor}

El modelo propuesto considera tres expresiones para representar el término fuente $\dot{Q}$ en función del grado de hidratación del cemento: el modelo propuesto por Azenha et al. (2009), el propuesto por Cervera et al. (1999) y el modelo propuesto por Jaafar et al. (2007). En todos los casos, los valores de los parámetros se obtienen de ensayos experimentales. Si consideramos el término fuente en 
función del grado de hidratación propuesto por Azenha et al. (2009), la expresión es implícita y puede escribirse como sigue:

$$
\dot{Q}=A f(\alpha) e^{-\left(E_{a} / R \phi\right)},
$$

donde $A$ es una constante cuyas unidades son $\mathrm{Wm}^{-3}, E_{a}$ es la energía de activación aparente en $\mathrm{kJ} \mathrm{mol}^{-1}, R$ es la constante de Boltzmann de valor $8.314 \mathrm{Jmol}^{-1} \mathrm{~K}^{-1}$, $\phi$ es la temperatura en $\mathrm{K}$, $\alpha$ es el grado de avance de la reacción de hidratación del cemento y $f(\alpha)$ es una función normalizada de expresión:

$$
f(\alpha)=c[\operatorname{sen}(\pi \alpha)]^{a} e^{(-b \alpha)}
$$

en la cual $a, b$ y $c$ son parámetros del cemento.

El término fuente para la ecuación del calor empleada por Cervera et al. (1999) parte de suponer la siguiente relación para el término de generación de calor en la masa de hormigón:

$$
\dot{Q}=Q_{\xi} \dot{\xi}
$$

El parámetro $Q_{\xi}$ puede obtenerse mediante un experimento adiabático, mientras que la evolución de la variable normalizada denominada grado de hidratación $\xi$ está dada por:

$\dot{\xi}=\frac{k_{\xi}}{\eta_{\xi_{0}}}\left(\frac{A_{\xi_{0}}}{k_{\xi} \xi_{\infty}}+\xi\right)\left(\xi_{\infty}-\xi\right) \exp \left(-\bar{\eta} \frac{\xi}{\xi_{\infty}}\right) \exp \left(-\frac{E_{a}}{R \phi}\right)$

donde $E_{a}$ es la energía de activación y $R$ es la constante de Boltzmann. Los restantes parámetros son: $A_{\xi 0}$ la afinidad química inicial, $\eta_{\xi_{0}}$ y $\bar{\eta}$ las viscosidades, y $k_{\xi}$ una constante del material. En dicha expresión interviene el valor final del grado de hidratación que se obtiene como:

$$
\xi_{\infty}=\frac{1.031 w / c}{0.194+{ }^{w} / c}
$$

siendo $w / c$ la relación agua-cemento de la mezcla. La expresión empleada por Jaafar et al. (2007) es explícita en el tiempo, y da la evolución de la temperatura. Para el caso del cemento, la evolución de la temperatura adiabática viene dada por:

$$
\phi_{a d}=K_{t}\left(1-e^{-\alpha t}\right)
$$

donde $\phi_{a d}$ es la temperatura adiabática, $K_{t}$ es la temperatura máxima en el hormigón en condiciones adiabáticas, $\alpha$ es un parámetro que regula cómo evoluciona la temperatura adiabática conforme transcurre el tiempo y posee unidades de $1 /$ tiempo y $t$ es el tiempo, medido en horas. El calor acumulado generado por la hidratación hasta el tiempo $t$ se expresa:

$$
Q=c_{e} \rho \phi_{a d}
$$

Con las expresiones (11) y (12) se puede escribir entonces:

$$
\dot{Q}=c_{e} \rho K_{t} \alpha e^{-\alpha t}
$$

Los valores $K_{t}$ y $\alpha$ se determinan para cada tipo de cemento.

\section{Modelo discreto}

La metodología numérica no se aplica a la forma fuerte del problema (ecuación diferencial de calor), sino a una forma débil obtenida a través del método de los residuos ponderados de Galerkin (Sergerlind, 1984). La no linealidad del problema, dado que el término fuente depende de la temperatura, se resuelve mediante una estrategia iterativa (Newton - Raphson). La discretización espacial se realiza por el método de elementos finitos y la discretización temporal, mediante un esquema implícito de integración (Backward-Euler) basado en diferencias finitas. Se propone una aproximación por elementos finitos lineales, triangulares para $2 \mathrm{D}$ y tetraédricos para $3 \mathrm{D}$, para el campo de temperaturas.

\section{Comparación con resultados experimentales}

El ensayo con el que se comparan los resultados del modelo es el realizado por Azenha et al. (2009) y consiste en el monitoreo del comportamiento termo-mecánico de un hormigón a temprana edad. Dicho experimento fue llevado a cabo mediante la colocación de sensores capaces de medir temperaturas y tensiones dentro de una probeta prismática simétrica de hormigón, ver Figura 1a. La misma se encontraba en un entorno controlado de temperatura y humedad relativa constantes.

La modelación numérica de la probeta se realizó aprovechando su doble simetría (Figura 1b), imponiendo condiciones de borde de flujo nulo en las caras de simetría y de convección en las superficies restantes, teniendo en cuenta el coeficiente de convección de cada material. Este y los restantes valores que gobiernan el modelo se 
encuentran indicados en la Figura 2.

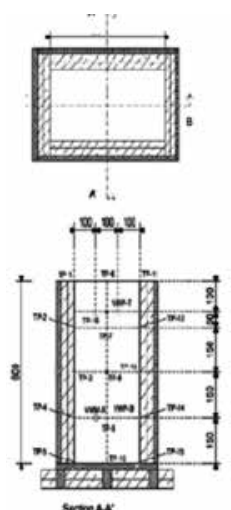

a)

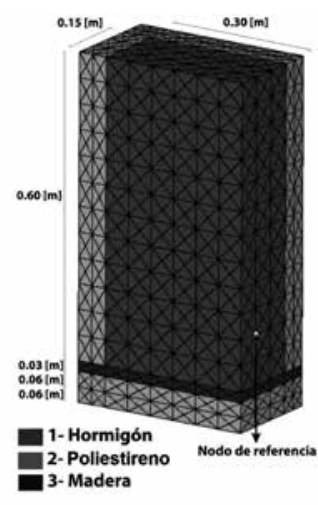

b)

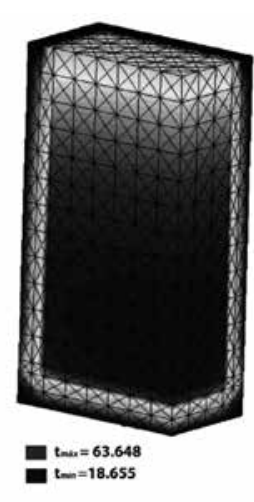

c)
Figura 1: a) Geometría del ensayo experimental, b) modelo de elementos finitos y c) distribución de temperaturas

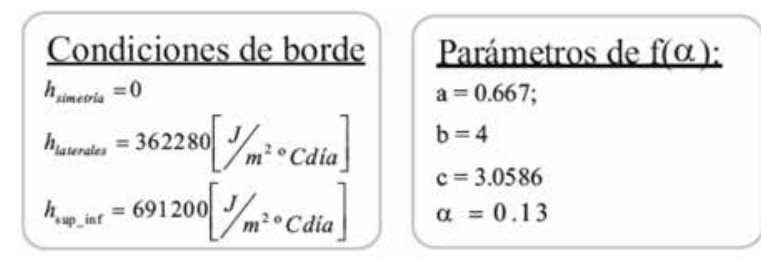

$$
\begin{array}{cc}
\text { Propiedades fisicas de los materiales } \\
k_{1}=22640\left[\mathrm{~J} / \mathrm{m}^{\circ} \mathrm{Cdia}\right] & \rho_{1} c_{1}=2400000\left[\mathrm{~J} / \mathrm{m}^{3 \circ} \mathrm{Cdia}\right] \\
k_{2}=3024\left[\mathrm{~J} / \mathrm{m}^{\circ} \mathrm{Cdia}\right] & \rho_{2} c_{2}=28400\left[\mathrm{~J} / \mathrm{m}^{1 \circ} \mathrm{Cdia}\right] \\
k_{3}=12960\left[\mathrm{~J} / \mathrm{m}^{\circ} \mathrm{Cdia}\right] & \rho_{3} c_{3}=854400\left[\mathrm{~J} / \mathrm{m}^{{ }^{\circ} \circ} \mathrm{Cdia}\right]
\end{array}
$$

Figura 2: Parámetros utilizados en el modelo numérico para condiciones de borde y propiedades de los materiales.

En el modelo numérico se consideró un término de generación de calor caracterizado por las ecuaciones (6) y (7) y cuyos parámetros están dados en la Figura 2. En la Figura 1c se muestra el campo de temperaturas para el tiempo $t=0.86$ días, obtenido mediante el modelo numérico desarrollado.

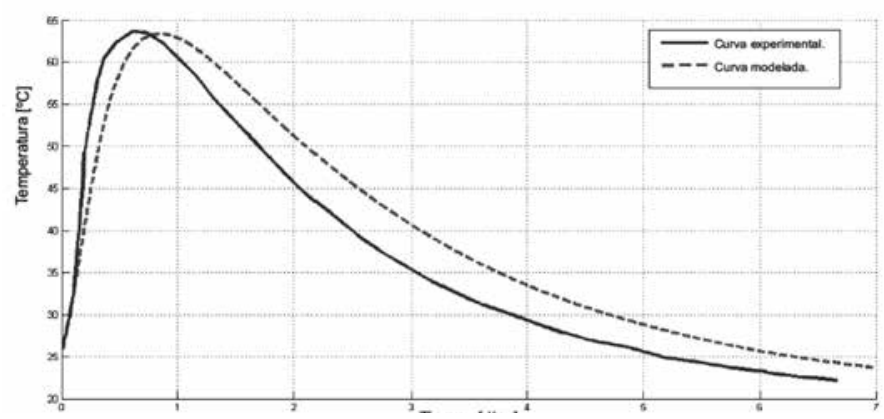

Figura 3: Curvas de evolución de temperatura del ensayo experimental y del modelo numérico
En la Figura 3 se comparan resultados del ensayo experimental con los del modelo numérico en términos de la temperatura en un nodo de referencia, en coincidencia con la ubicación de un sensor de temperatura (ver Figura 1b), apreciándose una correlación coherente entre ambos.

\section{Verificación del término fuente según Cervera et. al. (1999)}

Se compararon los resultados obtenidos mediante la simulación numérica con los estudios realizados por Cervera et al. (1999) en los que se ensayaron probetas cilíndricas de hormigón convencional (Tabla 1) y hormigón de cemento Portland C30 (Tabla 2). Se pueden observar las gráficas comparativas de evolución de la temperatura para el hormigón convencional (Figura 4) y cemento Portland C30 (Figura 5).

Tabla 1: Propiedades del hormigón convencional

\begin{tabular}{|l|l|}
\hline$w / c$ & 0.45 \\
\hline$\xi_{\infty}$ & 0.72 \\
\hline$k_{\xi} / \eta_{\xi 0}, 10^{7} 1 / \mathrm{h}$ & 0.28 \\
\hline$\eta$ & 5.30 \\
\hline$A_{\xi 0} / k_{\xi}$ & 0.50 \\
\hline$E_{a} / R, 10^{3}{ }^{\circ} \mathrm{K}$ & 5.00 \\
\hline$Q_{\xi}, 10^{5} \mathrm{~J} / \mathrm{m}^{3}$ & 2.02 \\
\hline
\end{tabular}

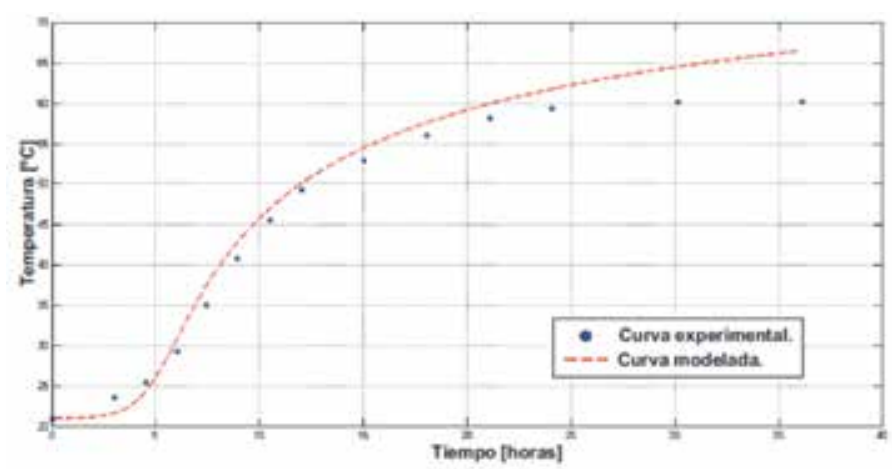

Figura 4: Evolución de temperatura para hormigón convencional 
Tabla 2: Propiedades del Cemento Portland 30

\begin{tabular}{|l|l|}
\hline$w / c$ & 0.50 \\
\hline$\xi_{\infty}$ & 0.75 \\
\hline$k_{\xi} / \eta_{\xi 0}, 10^{7} 1 / \mathrm{h}$ & 0.14 \\
\hline$\eta$ & 7.00 \\
\hline$A_{\xi 0} / k_{\xi}$ & $10^{-4}$ \\
\hline$E_{a} / R, 10^{3} \mathrm{~K}$ & 4.00 \\
\hline$Q_{\xi}, 10^{5} \mathrm{~J} / \mathrm{m}^{3}$ & 1.58 \\
\hline
\end{tabular}

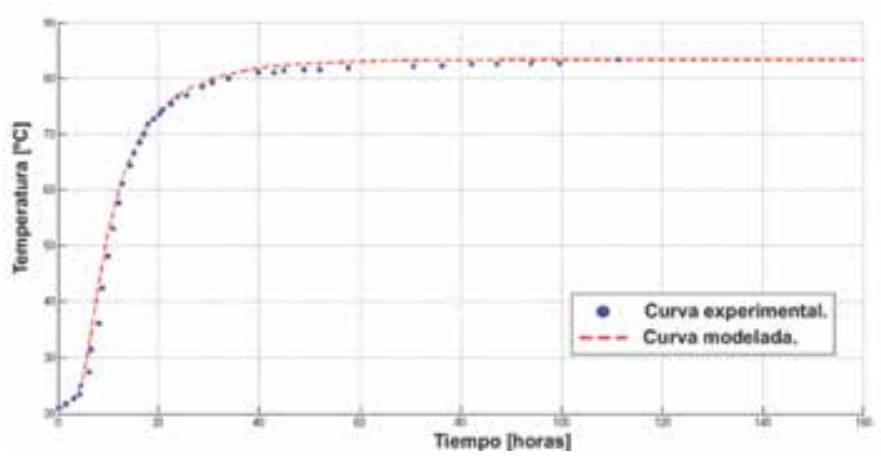

Figura 5: Evolución de temperaturas para cemento Portland C30

\section{Estudio de una presa de hormigón compactado a rodillo}

Con el fin de validar la metodología en cuanto a la construcción de estructuras realizadas por etapas, se analiza una presa real de hormigón compactado a rodillo (HCR) de $169 \mathrm{~m}$ de altura (ver Figura 6). Los datos de la estructura se toman del trabajo de Jaafar et al. (2007), del cual también se toman sus resultados para comparar con el modelo de este trabajo. Los parámetros usados en la fórmula del término fuente, propios del hormigón, son $\mathrm{K}_{\mathrm{t}}=17^{\circ} \mathrm{C}$ y $\alpha=0.0183$. El incremento de tiempo de análisis es $\Delta \mathrm{t}=3 \mathrm{~h}$. Si bien en las estructuras de HCR, el material es colocado en capas horizontales de $30 \mathrm{~cm}$, se trabajó con capas de $5 \mathrm{~m}$ aplicadas cada 20 días para disminuir el trabajo computacional.

La construcción de la presa finalizó a los 22.5 meses de comenzada la obra. Para el análisis, la presa fue dividida en 34 etapas. Se utilizó una malla de elementos finitos triangulares (Figura 7) adecuada para la geometría (Figura 6), con un total de 1783 nodos y 3296 elementos. Las condiciones de borde se observan en la Figura 8 y los datos de los materiales en la Tabla 3. Tanto la temperatura ambiente como la temperatura de colocación se observan en la Figura 9.

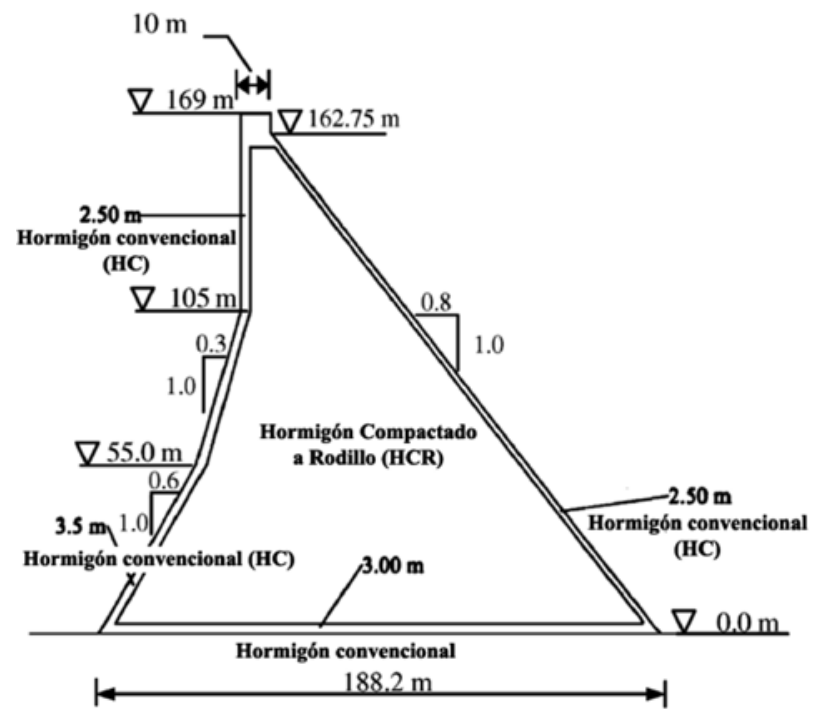

Figura 6: Sección de la presa, geometría y materiales

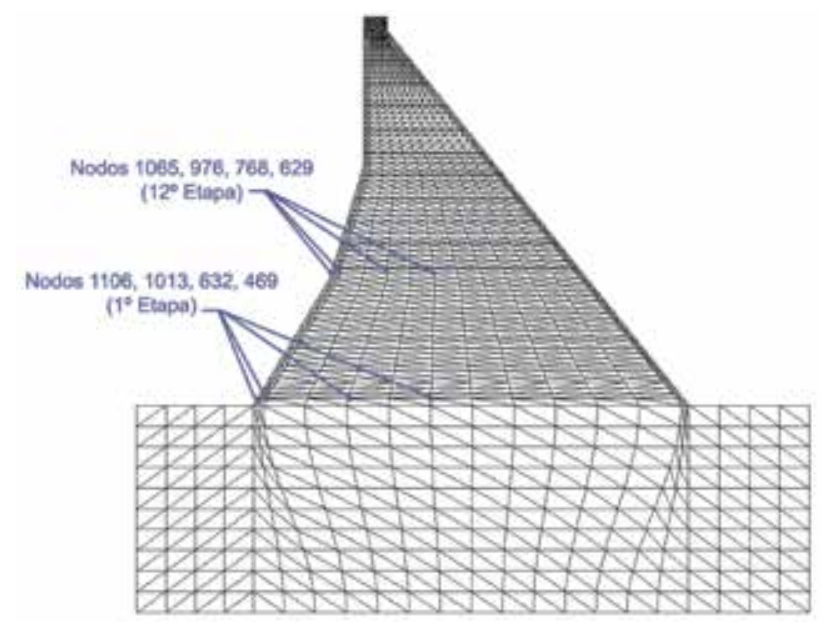

Figura 7: Malla de elementos finitos, nodos seleccionados para comparar resultados

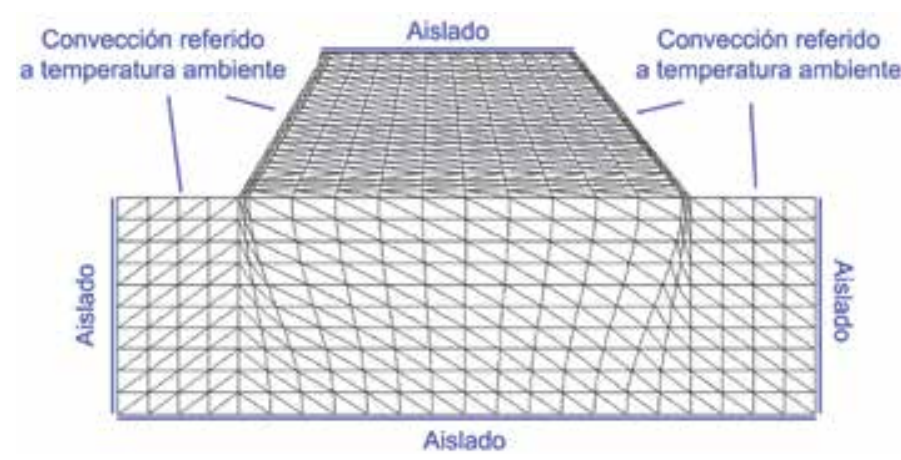

Figura 8: Condiciones de borde 


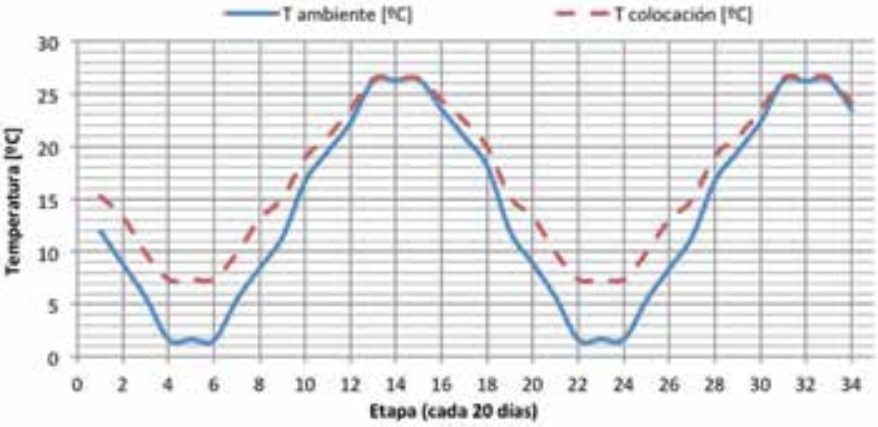

Figura 9: Temperatura ambiente y de colocación
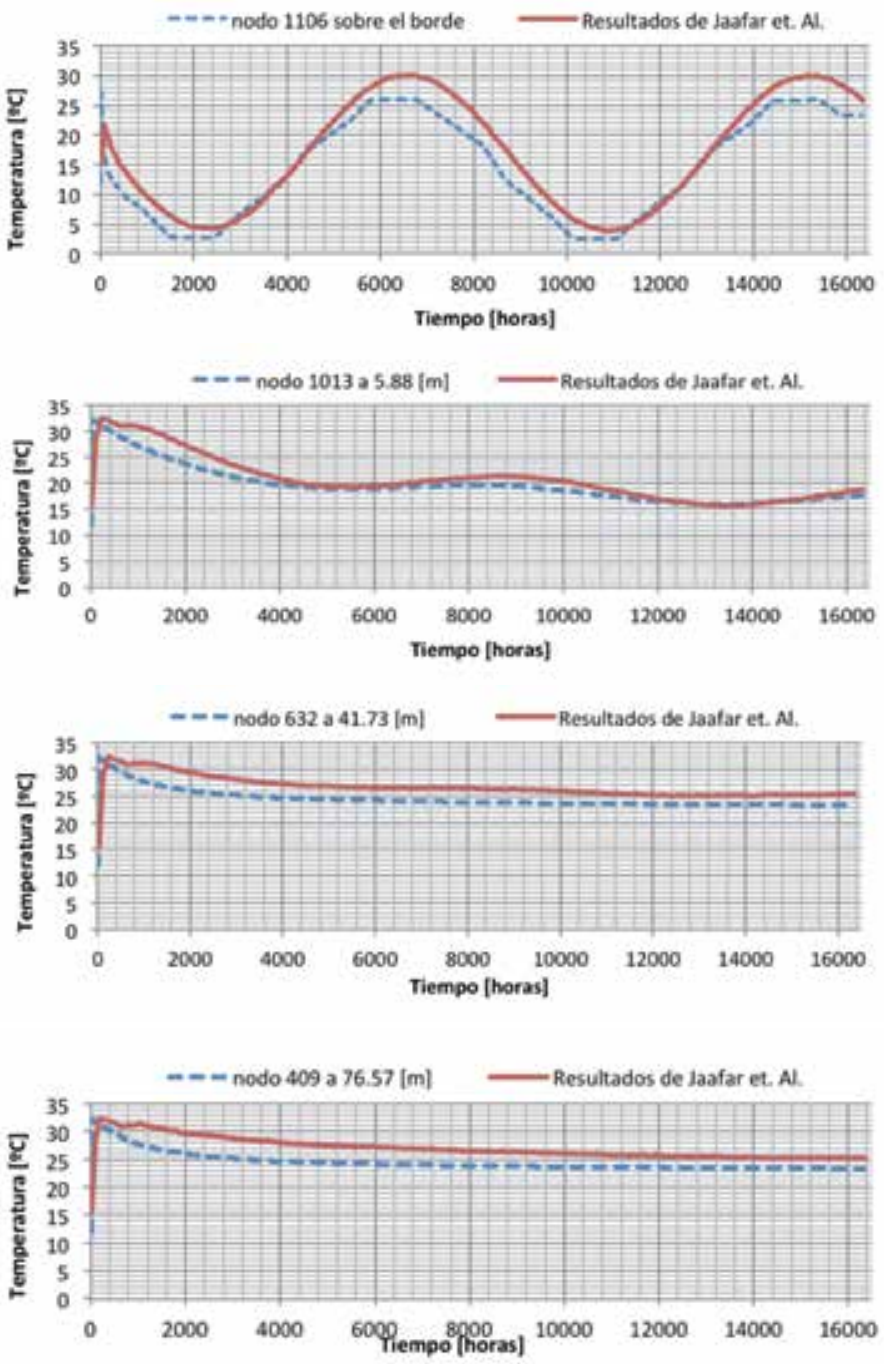

Figura 10: Comparación de resultados en los nodos seleccionados de la etapa 1

Las Figuras 10 y 11 muestran la evolución de la temperatura en el tiempo para nodos seleccionados dentro de la presa (Figura 7). La comparación de resultados se realiza respecto al trabajo de Jafaar et al. (2007), tanto para nodos de la etapa 1 como para nodos de la etapa 12.
Tabla 3: Propiedades de los materiales de la presa: roca de fundación, hormigón convencional HC y hormigón compactado a rodillo HCR

\begin{tabular}{|l|c|c|c|}
\hline \multirow{2}{*}{ Propiedades } & \multicolumn{3}{|c|}{ Materiales } \\
\cline { 2 - 4 } & Roca & HC & HCR \\
\hline $\begin{array}{l}\text { Coeficiente de conducción } k, \\
\mathrm{kcal} / \mathrm{mh}^{\circ} \mathrm{C}\end{array}$ & 1.3 & 1.81 & 1.81 \\
\hline $\begin{array}{l}\text { Coeficiente de convección } h, \\
\mathrm{kcal} / \mathrm{m}^{2} \mathrm{~h}^{\circ} \mathrm{C}\end{array}$ & 10.0 & 10.0 & 10.0 \\
\hline Calor específico $c_{e}, \mathrm{kcal} / \mathrm{kg}^{\circ} \mathrm{C}$ & 0.22 & 0.22 & 0.22 \\
\hline Densidad $\rho, \mathrm{kg} / \mathrm{m}^{3}$ & 2710 & 2400 & 2400 \\
\hline
\end{tabular}
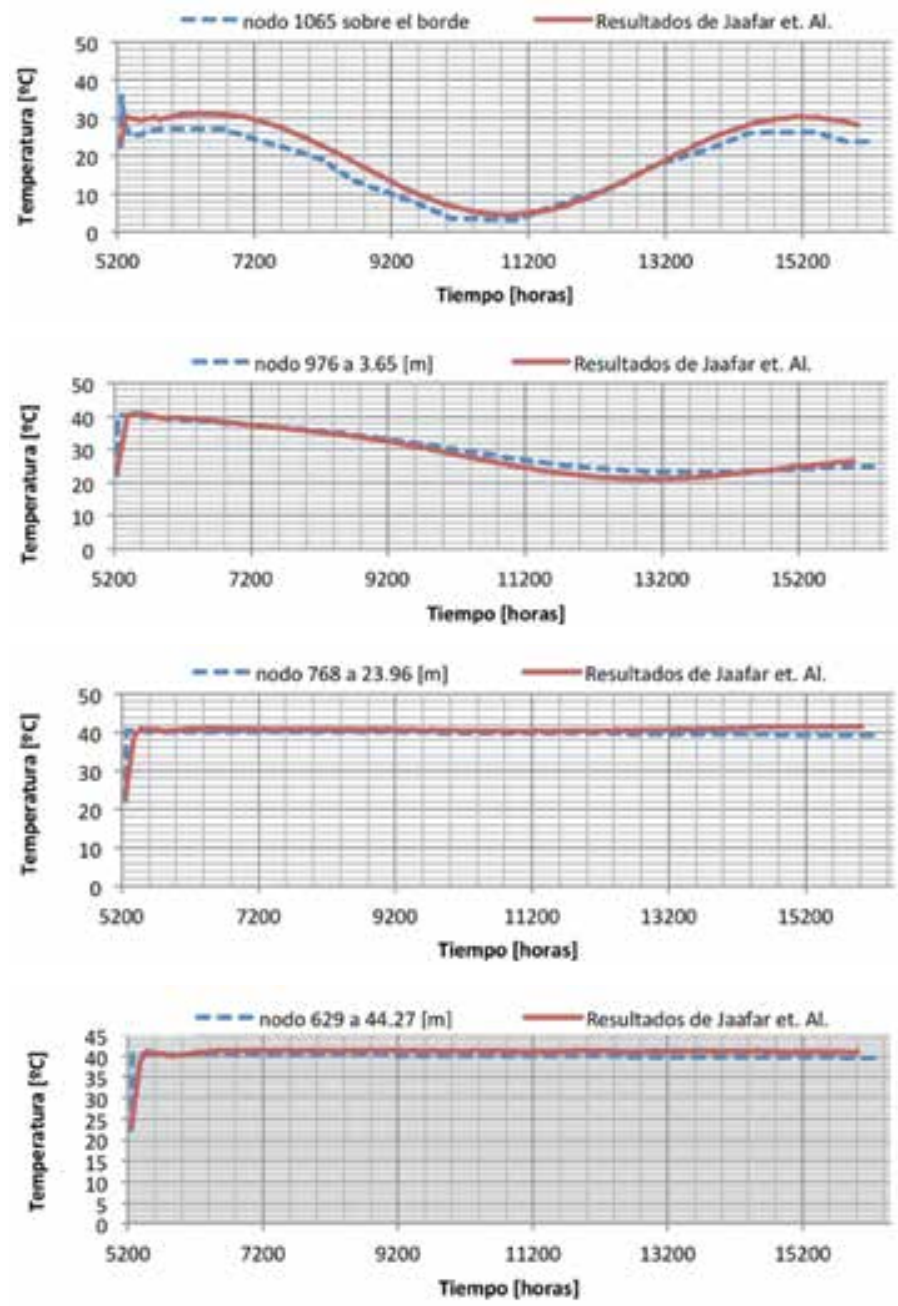

Figura 11: Comparación de resultados en los nodos seleccionados de la etapa 12

Se observa que en las proximidades de los bordes de la presa la temperatura tiende a asemejarse a la temperatura ambiente, mientras que en el cuerpo de la presa tiende a condiciones adiabáticas, sin influencia de las variaciones 
de la temperatura ambiente.

En las Figuras 12 y 13 se observan los mapas de temperaturas obtenidos por Jaafar et. al. (2007) y los que resultaron de este análisis. La distribución de la temperatura es semejante, coincidiendo los sectores de mayores valores en el centro de la presa.

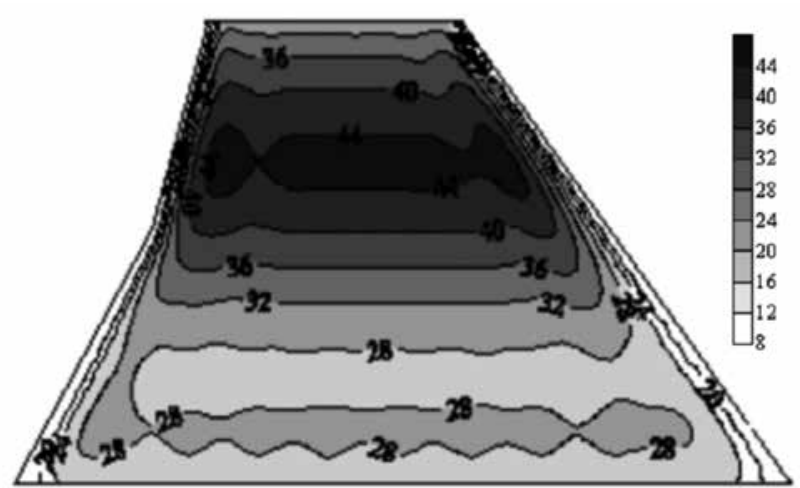

(a)

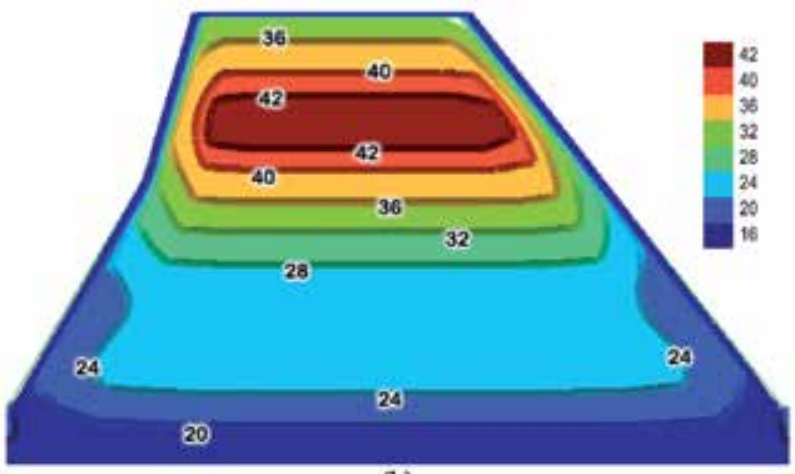

(b)

Figura 12: Comparación de mapas de temperatura de la presa en la etapa 20, a) Jaafar et al. (2007) y b) análisis presente

Se observa que la influencia de la temperatura ambiente cumple un papel fundamental a la hora de planificar el hormigonado por etapas. Las mayores temperaturas son alcanzadas en las etapas 14 y 32 donde la temperatura ambiente es máxima. Esto se puede corroborar en la Figura 9. Esas temperaturas máximas se acumulan principalmente en el centro de la presa, al tener este sector condiciones cuasi-adiabáticas.

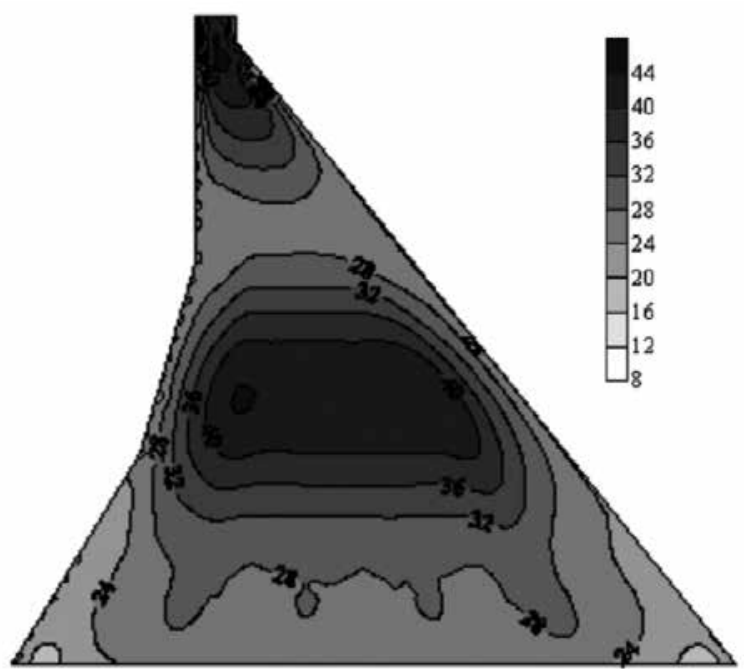

(a)

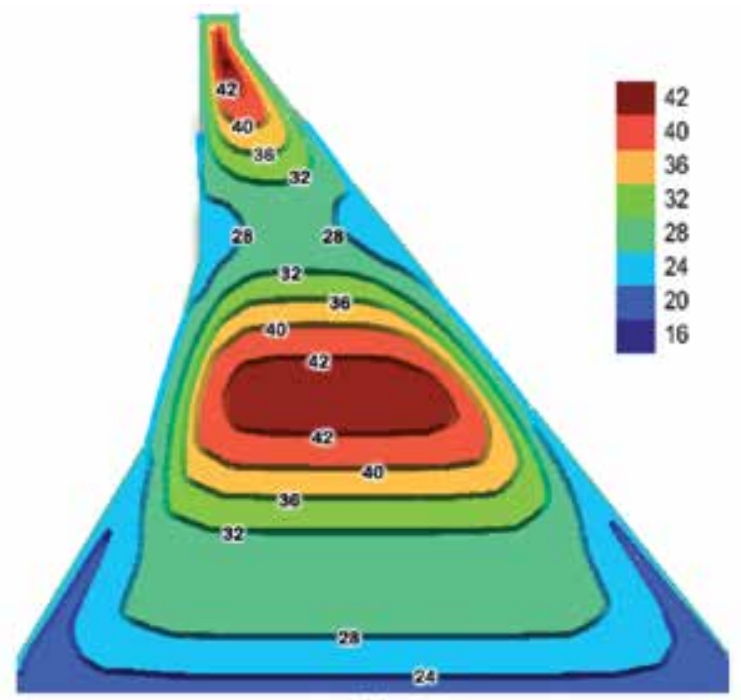

(b)

Figura 13: Comparación de mapas de temperatura de la presa en el fin de su construcción (etapa 34), a) Jaafar et al. (2007) y b) análisis presente

\section{Conclusiones}

Se desarrolló un modelo numérico capaz de predecir la evolución de temperatura en distintas estructuras de hormigón debido a la hidratación del cemento. El modelo permite encarar problemas de transferencia de calor con distintas condiciones iniciales y de contorno. A su vez, se analizan estructuras ejecutadas por el método del hormigonado por etapas. Se resuelve el problema térmico para las distintas etapas, simulando la colocación de nuevas capas y actualizando las condiciones de borde a medida que avanza la construcción. Se concluye que los resultados 
muestran una buena correlación con los obtenidos a partir de ensayos experimentales y trabajos de otros autores, lo cual permite validar el modelo propuesto.

\section{Referencias}

Azenha, M. and Faria, R. (2008). Temperatures and stresses due to cement hydration on the $\mathrm{R} / \mathrm{C}$ foundation of a wind tower - A case study. Engineering Structures 30(9), 2392-2400

Azenha, M., Faria, R. and Ferreyra, D. (2009). Identification of early-age concrete temperatures and strains: Monitoring and numerical simulation. Cement and Concrete Composites 31(6), 369-378

Cervera, M., Oliver, J. y Prato, T. (1999). Un modelo termoquímico-mecánico de hormigón a tempranas edades. Aplicación a la construcción de presas de HRC. Monografía CIMNE, 51
Crespo, M.D. (2011). Comportamiento del hormigón armado a tempranas edades. Efectos estructurales de las deformaciones impuestas impedidas. Tesis Doctoral, Universitat Politècnica de Catalunya

Incropera, F.P. and DeWitt, D.P. (2002). Fundamentals of heat and mass transfer. New York: John Wiley \& Sons

Jaafar, M.S., Bayagoob, K.H., Noorzaei J. and Thanoon Waleed, A.M. (2007). Development of a finite element computer code for thermal analysis of roller compacted concrete dams. Advances in Engineering Software 38(11), 886-895

Sergerlind, L.J. (1984). Applied finite element analysis. New York: John Wiley and Sons 